\title{
The Effect of Viscosity on Particle Suspension in an Aerated Stirred Vessel with Different Impellers and Bases
}

\section{S. IBRAHIM1 AND A. W. NIENOW2}

1 Department of Civil Engineering, Faculty of Engineering, University of Malaya, Kuala Lumpur, Malaysia

2Department of Chemical Engineering, University of Birmingham, Birmingham, U.K.

Introduction

This article is the fourth in a series mainly devoted to characterizing the suspension of particulate solids in a baffled stirred vessel, diameter $T(1 / 40.29 \mathrm{~m})$, using a variety of traditional and newer impellers. The impellers used in this study are described in

Please refer to the full text

Table I. Newtonian fluids with a range of viscosities from $1 \mathrm{mPas}$ (water) to 1 Pas (corn syrup) were employed as well as three different vessel bases, as shown in Figure 1. In the first article (Ibrahim and Nienow, 1995), the unaerated power characteristics and flow patterns of the impellers in the different fluids were established covering Reynolds numbers from $40<R e<5 \_105$. In the second article (Ibrahim and Nienow, 1996), suspension in the turbulent region was studied with each of the impellers and bases. For this work, Ballotini lead glass beads, diameter, $\mathrm{dP}$ $(1 / 4655 \mathrm{~mm})$, and density, qL (1/42950 kg=m3), at a concentration of $0.5 \mathrm{wt} . \%$ were used. This low concentration made the visual assessment of the minimum speed required to achieve the just-suspended condition, Nus, based on the Zwietering criterion (Zwietering, 1958), very easy. It was then assumed, based on extensive earlier work (Zwietering, 1958; Nienow, 1968; Chapman et al., 1983a), that the observed Nus at this concentration could be generalized for concentration up to about 


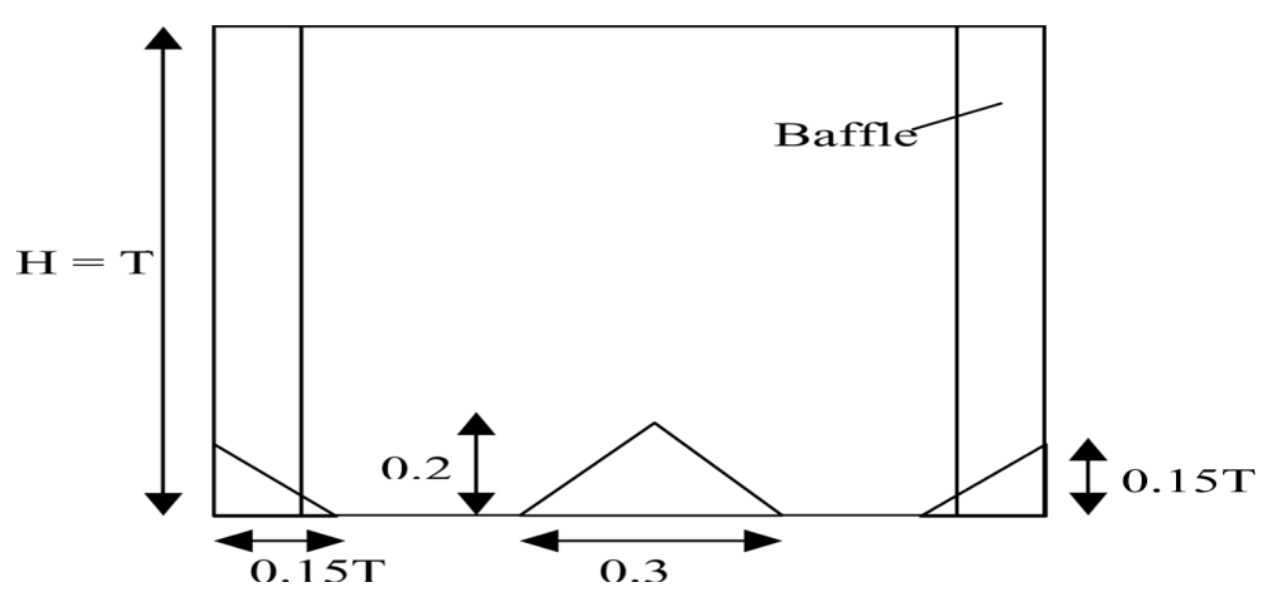

Figure 1. Vessel ( $T 1 / 40.29 \mathrm{~m})$ with four $0.1 \mathrm{~T}$ baffles and the cone-and-fillet base; the removal of the latter gives the flat base.

$20 \mathrm{wt} . \%$ by determining the dimensionless suspension parameter, $\mathrm{S}$, in the equation (Zwietering, 1958):

Please refer to the full text

where $X$ is the solid concentration ( $\mathrm{kg}$ solids=kg liquid_100). The S parameter depends on the impeller type and the $\mathrm{D}=\mathrm{T}$ and $\mathrm{C}=\mathrm{T}$ ratios (for vessels where the liquid height, $\mathrm{H}^{1} / 4 \mathrm{~T}$ ) but is independent of scale. This equation is still recommended in the latest major text on mixing (Atiemo-Obeng et al., 2004). It has been confirmed again in 2008 using a different measurement technique to the visual observation generally employed (Selima et al., 2008). The third article (Ibrahim and Nienow, 1999) extended the study to the higher viscosity fluids.

In general, these studies showed that the down-pumping axial flow impellers (HE3 and MFD) required the lowest mean specific energy dissipation rates, (ет)JS $\mathrm{W}=\mathrm{kg}$, to achieve Nus provided the flow was in the turbulent or high transitional Re regime, i.e., for water and 0.01 Pas corn syrup. If the bottom was flat, this occurred at $\mathrm{D}=\mathrm{T} \_0.4$ for both types, although (ет)」s for the HE3 was relatively insensitive to $D=T$ compared to the MFD for which it increased rapidly at greater $D=T$ values. The change to the cone-and-fillet base greatly reduced (ет)лs for both impellers at the same $D=T$ but was even lower with the HE3 at $D=T \_0.3$. The radial flow Rushton turbines, RT (as commonly reported), and the dual InterMIGs both required significantly higher values of (ет)лs. However, at the highest viscosity of 1 Pas (Re_200), the discharge from all the impellers became approximately radial and all the largest $D=T$ impellers became the most efficient, including the 
InterMIGs, and in general, (ет)ıs was significantly higher than in the higher $\mathrm{Re}$ situations. In most cases at all viscosities, (ет)лs was minimized by positioning the impeller clearance at $\mathrm{C}=\mathrm{H}^{1} / 41=6$, although clearances of $1=4$ and $1=3$ were also studied. The benefits of using low clearances were also shown by Armenente and Nagamine (1998).

This article builds on the earlier work to consider the problem of suspending the same solids in water and 0.01 Pas and 0.1 Pas corn syrup to give $\_2000<R e<\_105$ with both the flat and the cone-and-fillet bases (Figure 1) with gassing rates up to $3 \mathrm{vvm}$.

\section{Literature}

Recent work on solid suspension has indicated an additional factor that needs consideration under turbulent conditions in relation to Nus, namely the stress as a result of friction between the particle and the base that depends on particle shape (Thorpe and Stevenson, 2003). A similar effect is found with bases of different surface roughness, although in that case it is also dependent on how the roughness affects the local boundary layer and turbulence (Ghionzoli et al., 2007). However, the effect is relatively small and in all four comparative studies, the base is the same and rather smooth, being made of Perspex.

There has been very little additional relevant work on the effect of viscosity. Wu et al. (2001) considered the effect of shear thinning behavior by the addition of the soluble viscosifying polymer Carbopol. They particularly connected the change of Nus with reductions in the impeller pumping ability as the fluid became more viscous.

The effect was very complex because although the flow required to suspend particles was lowered, the speed at which they settled was also reduced. The overall effect was initially to lower Nus slightly and then to increase it. At a certain concentration of Carbopol, the particles did not settle at all, so agitation was not required!

Once the effect of gas sparging requires consideration, the literature on solid suspension under these conditions has to be reviewed. In three-phase systems, the impeller has to simultaneously disperse gas bubbles and suspend solid particles. The challenge in selecting an impeller for performing the dual function is the conflicting design requirements for solid suspension and gas dispersion. Much of the literature has been summarized for the turbulent flow regime by Nienow and Bujalski (2002). Under these conditions, in general, gas sparging can be considered to have two effects. First, in general, it leads to gas-filled cavities forming behind impeller blades, which lowers both the power input and the pumping rate of the impeller (Warmoeskerken et al., 1984). These changes generally lead to an increase in 
Nusg with increasing gas flow rate compared to Nus (Chapman et al., 1983b; Nienow et al., 1986). Provided the flow rate at different scales is expressed in flow rate=fluid volume in the reactor (for example, QGv vvm), the same relationship was found to apply for geometrically similar vessels from _ 0.3 to _ $1.8 \mathrm{~m}$ diameter.

Second, the sparged gas tends to enhance an upward liquid flow associated with the rising bubbles. The latter effect is particularly significant for MFD impellers where, if the gas goes straight from the sparger to the impeller (direct loading), suspension generally cannot be achieved, and with propellers (Subbarao and Taneja, 1979) and small $D=T$ and low solidity MFD impellers (Chapman et al., 1983), there is a sudden catastrophic loss of solid suspension with gas sparging. Wong et al. (1987) particularly proposed the use of a Rushton turbine over an MFD impeller for high gassing rates. If, on the other hand, the sparged gas is well dispersed off the sparger, being driven downwards from the impeller to the base of the vessel (indirect loading), suspension is possible. Indeed, at very low gas flow rates, Nus and (ет)лs may both be greater than their equivalent values under gassed conditions, Nısg and (ет) Jsg, because the bubbles, as they approach the base, disrupt the boundary layer in the region (Chapman et al., 1983b; Frijlink et al., 1990). To overcome the effect of rising bubbles, it is best to use relatively large $D=T$ impellers of high solidity ratio (projected horizontal area of blades=horizontal area swept out by impeller rotation), such as 6MFD (Chapman et al., 1983b) or wide-blade hydrofoils (Nienow and Bujalski, 2002).

An alternative approach is to use an up-pumping, axial flow impeller, so that the upward liquid flow in the wake of rising bubbles and the flow generated by the impeller combine to give very vigorous liquid recirculation around the impeller and across the base. As the gas flow rate increases, so does the bubble entrained flow and that recirculating across the base, and Nusgand (ет) Jsg both increase only relatively slowly. These effects are found with large $D=T$, high solidity ratio impellers whether 6MFU (Chapman et al., 1983b) or particularly wide-blade hydrofoils (Nienow and Bujalski, 2002). At the highest gas flow rates tested, the up-pumping impellers had the lowest (ет).Jsg (Chapman et al., 1983b; Nienow and Bujalski, 2002, 2004), and although generally supported by Frijlink et al. (1990), the latter suggested that Rushton turbines were more efficient at high solids loading.

For both T=2, 6MFU and T=2, 6MFD, Chapman et al. (1983b) and Nienow et al. (1986) found that provided the flow rate at different scales was expressed as QGv vvm, simple equations relating Nusg to Nıs and QGv applied to geometrically similar vessels from_ 0.3 to _ $1.8 \mathrm{~m}$ diameter.

To the authors' knowledge, work on three-phase systems with fluids of 
significantly higher viscosity than water has not been reported, and it is that gap that this article begins to fill. However, the range of impellers studied in any depth compared to those reported in the three first articles of this series has been reduced somewhat. This reduction is based on two observations. First, for all the different solid sizes, densities, and concentrations used, unless the gas was dispersed, the work of Chapman et al. (1983b), Nienow et al. (1986), and Nienow and Bujalski (2002) all showed that suspension could not be achieved. For the impellers tested by them that were also used in the three earlier articles on which this one builds, the larger impellers of size _ $\mathrm{T}=2$ dispersed a particular airflow rate at lower (ет)g values than the smaller impellers. Since larger impellers, as shown above, became progressively more effective at suspending solids as the viscosity of the fluid increased, it was decided to concentrate on the _ $\mathrm{T}=2$ impellers in the work on three-phase systems reported here. In addition to measuring the power input, NJSg and (eT)Jsg, for the glass Ballotini in three-phase mixing in water and corn syrup solutions of 0.01 Pas and 0.1 Pas under ungassed and gassed conditions up to $3 \mathrm{vvm}$, a description of the particle distribution on the tank base was made in order to provide insight into how the power input and flow regime related to particle movement and the points on the base where suspension occurred.

\section{Materials and Methods}

All the experiments were conducted in a $0.29 \mathrm{~m}$ diameter Perspex vessel with an outer jacket filled with water to allow an undistorted view of the vessel contents. The vessel was mounted on an air bearing and connected to a load cell to facilitate power measurements. The shape of the base was either flat or modified with a cone and fillet (Figure 1). Further details on the "cone-and-fillet" base are given elsewhere (Ibrahim and Nienow, 1996). The impellers used are given in Table I and their details have been published before (Ibrahim and Nienow, 1995). In the earlier work on solid suspension, an impeller clearance $\mathrm{C}=\mathrm{T}^{1} / 41=6$ had generally been shown to give the lowest (eт)Js. However, since work on gas dispersion had shown instabilities with such clearances (Chapman et al., 1983b; Nienow et al., 1986), for the three-phase work, an impeller clearance of $\mathrm{C}=\mathrm{T}^{1 / 1} 41=4$ was used in all cases.

Gas was introduced via a point or ring sparger, $D_{s}{ }^{1} / 40.135 m\left(D_{s}=T^{1} 1 / 40.47\right)$, with the flat-bottomed tank but only the ring sparger was used with the "cone-and-fillet" base because the point sparger could not be placed at the center due to the presence of the cone. Flow rates ranged from 0.25 up to $3 \mathrm{vvm}$ (QGv), and the equivalent flow rates in $\mathrm{m}_{3}=\mathrm{s}$ are shown in Table II. The fluids used were water and $0.01 \mathrm{Pas}$ and 0.1 Pas corn syrup solutions. With the cone-and-fillet base, only water was used. 
The solids were spherical blue lead glass Ballotini beads ( $\mathrm{dP}^{1} 1 / 4655 \mathrm{~mm}$, $\mathrm{qs}^{1} / 42950 \mathrm{~kg}=\mathrm{m}_{3}$ ) at $0.5 \mathrm{wt}$ \% for reasons explained above. In every run, complete gas bubble dispersion occurred at $\mathrm{N}<\mathrm{N}$ ssg, as reported by Nienow and Bujalski (2002). In the case of down-pumping impellers, when the dispersion of gas bubbles can be unstable and asymmetric, at the NJsg reported here, the dispersion of bubbles was still deemed acceptable. Nus and Nusg were determined visually using Zwietering's (1958) criterion of "just-suspension" by observing the particles on the base of the tank through a mirror placed at an angle underneath it. Representative repeat measurements gave variation in Nus or Nusg of less than $5 \%$ in water and less than $10 \%$ in corn syrup.

\section{Results and Discussions}

\section{Flat Base Tank}

Three-Phase Suspension with Small 6MFD ( $\left.D=T^{1} 1 / 40.38\right)$ and All HE3 Impellers These impellers are grouped together because they show the same problem of asymmetry and instability associated with gas dispersion, which is particularly severe with these impellers due to a small number of blades in the case of the HE3 and small $D=T$ for the 6MFD. At low speeds, when the impeller was flooded even at very low gas flow rates, gas bubbles were concentrated near the impeller, and particles on the base were swept to the corner of the tank, where they remained stationary (Figure 2(a)). Increasing the speed caused an outward spread of the bubbles, and when clinging cavities were observed, a fairly even symmetric dispersion occurred. Full text is available at :

http://www.tandfonline.com/doi/abs/10.1080/00986440903245914\#.UwFf4vu9gtE 